Research Article

\title{
Programmed Cell Death Protein Ligand 2 Is a Potential Biomarker That Predicts the Efficacy of Immunotherapy
}

\author{
Aoyun Wang $\mathbb{D}^{1,2}$ Han Chu $\mathbb{D}^{1},^{3}$ Zheng Jin $\mathbb{D}^{4},{ }^{4}$ Qingzhu Jia $\mathbb{D}^{1,2}$ and Bo Zhu $\mathbb{D}^{1,2}$ \\ ${ }^{1}$ Institute of Cancer, Xinqiao Hospital, Third Military Medical University, Chongqing 400037, China \\ ${ }^{2}$ Chongqing Key Laboratory of Tumor Immunotherapy, Chongqing 400037, China \\ ${ }^{3}$ Center of Growth, Metabolism and Aging, Key Laboratory of Bio-Resources and Eco-Environment, College of Life Sciences, \\ Sichuan University, Chengdu 610064, China \\ ${ }^{4}$ Research Institute, GloriousMed Clinical Laboratory (Shanghai) Co., Ltd., Shanghai 201318, China
}

Correspondence should be addressed to Qingzhu Jia; jiaqingzhu0801@outlook.com and Bo Zhu; bo.zhu@tmmu.edu.cn

Received 6 September 2021; Accepted 16 October 2021; Published 31 October 2021

Academic Editor: Jie Mei

Copyright (C) 2021 Aoyun Wang et al. This is an open access article distributed under the Creative Commons Attribution License, which permits unrestricted use, distribution, and reproduction in any medium, provided the original work is properly cited.

Immune checkpoint inhibitor (ICI) responses vary, and biomarkers for predicting responders are urgently needed. Growing evidence points to the association between programmed cell death protein ligand 2 (PDL2) and ICI benefits, while clinical evidences were lacking. Thus, we consolidated five public ICI-treated cohorts to investigate the association between PDL2 expression and ICI treatment prognosis. Immune cell signatures and IFN- $\gamma$ signatures are investigated in The Cancer Genome Atlas (TCGA) dataset and later in ICI-treated cohorts to explore the association between PDL2 and antitumor immunity in the tumor microenvironment (TME). We found that immune cell signatures and IFN- $\gamma$ signatures were enriched in the PDL2high group in TCGA pooled cohorts and most cancers. Consistently, in ICI-treated cohorts, patients with high PDL2 expression experienced longer overall survival time (OS) and were more likely responsive to ICIs than patients with low PDL2 expression. Immune cell scores of the high PDL2 expression patients were significantly higher $(P<0.05)$ than those of the low PDL2 expression patients in ICI-treated cohorts. In conclusion, our findings suggest that PDL2 is a potential predictive biomarker for ICIs.

\section{Introduction}

ICIs, which combine the proteins of programmed cell death protein 1 (PD-1) axis or cytotoxic T lymphocyte-associated protein 4 (CTLA-4), are capable of improving the clinical prognosis of patients with various forms of cancers [1-4]. However, the low response rate in unselected populations limits their clinical efficacy. The active PD-1 signature in the tumor microenvironment is attributable for the escape of tumor immunity $[5,6]$. Binding of PD-1 to its ligands, programmed cell death protein ligand 1 (PDL1) and PDL2, can result in a failed CD8+ T cell activation, which weakens the signals produced by $\mathrm{CD} 28$ and $\mathrm{T}$ cell receptor $[7,8]$. PD1 is mainly expressed by activated T cells, and PDL1 appears on antigen-presenting cells (APCs) or tumor cells [9]. Thus, PDL1 immunohistochemistry (IHC) is now the mainstay for the clinical screening of ICI responders from nonresponders.
However, in previous studies, the responses to ICI treatment were inconsistent; some PDL1-positive patients responded poorly, while some PDL1-negative patients had considerable response rates [10-12]. Even in many clinical trials, no correlation was observed between the PDL1 expression and ICI treatment benefits [13-15]. Therefore, new predictive biomarkers are urgently needed for patients with cancer.

PDL2 is the other ligand for PD-1. Unlike PDL1, PDL2 was initially only observed in APCs [6]. Recently, after microenvironmental stimulation, researchers found that many immune cells and tumor cells could express PDL2 [16-19]. PDL2 is also considered a potential therapeutic target in prostate cancer [20]. One clinical study showed incomplete expression between the PDL1 and PDL2 in head and neck squamous cell carcinoma patients [21]. Consistently, animal models indicated combined anti-PDL1 and anti-PDL2 could abolish the tolerance after single anti- 
PDL1 treatment $[22,23]$. Vivo trails show PDL1-specific and PDL2-specific T cells represent different T cell antigens [24]. However, the investigation between PDL2 and the prognosis of patients across multiple cancers receiving ICI treatment was still lacking, and the possible behavior of PDL2 in the TME remains unclear. Therefore, we conducted this study using data collected from RNA-seq and clinical information of published ICI cohorts to explore the effect of PDL2 expression on the prognosis of patients receiving ICI treatment for different cancers. We also used TCGA database to explore the characteristics of PDL2 in the TME.

\section{Materials and Methods}

2.1. Public Data Collection. The mRNA expression profiles of TCGA patients were downloaded from the Genomic Data Commons (https://portal.gdc.cancer.gov/) using the R package TCGAbiolinks (https://bioconductor.org/packages/ release/bioc/html/TCGAbiolinks.html). The samples that had sequencing quality evaluated as " $\mathrm{A}$ " were retained. GRCh38.homo (https://asia.ensembl.org/index.html) was used for Gene ID conversion; the mRNA data of nonprotein-coding genes were excluded. Considering that LAML is not a solid tumor, patients with LAML were excluded. In each type of cancer, we divided samples into two groups using the median of PDL2 expression.

Three melanoma cohorts (Gide cohort: $n=73$; Liu cohort: $n=121$; and Van Allen cohort: $n=41$ ), a bladder cancer cohort (Mariathasan cohort: $n=348$ ), and a clear cell renal cell carcinoma cohort (Miao cohort: $n=33$ ) with detailed clinical information, response data, and mRNAseq data from published researches were analyzed [25-29]. All patients were treated with anti-PD-(L)1, anti-CTLA-4, or anti-PD-(L)1 combined with anti-CTLA-4. The efficacy of antitumor immunotherapy was evaluated by the Response Evaluation Criteria in Solid Tumors (RECIST) version 1.1. Patients who achieved complete response (CR) and partial response $(\mathrm{PR})$ or had stable disease $(\mathrm{SD})$ that lasted for $>6$ months were considered to have response to ICI treatment. All other patients were considered to have no response to ICI treatment.

2.2. Gene Set Enrichment Analysis. We performed GSEA (https://www.gsea-msigdb.org/gsea/index.jsp) to detect the distribution of immune genes in TCGA cohorts. CD8+ T cell signature, DC signature, Th1 signature, and IFN- $\gamma$ gene signatures were collected from published studies [30-33]. The immune infiltration scores of three cell types in each ICI sample were calculated using Single Sample Gene Set Enrichment analysis (ssGSEA) of GSVA of $\mathrm{R}$ package (http://bioconductor.org/packages/release/bioc/html/GSVA .html). The fragments per kilobase of transcript per million mapped reads (FPKM) of protein-coding genes between the high PDL2 expression and low PDL2 expression groups were analyzed in each cancer and TCGA pooled cohort. The results were considered statistically different when normalized $P$ values $(P)$ were $<0.05$.
2.3. Statistical Analysis. Univariate Cox regression was used to analyze whether high PDL2 expression is protective $(0<$ hazard ratio $(\mathrm{HR})<1)$ or increases the risk $(\mathrm{HR}>1)$ regarding the prognosis of ICI treatment. The overall survival (OS) was assessed and compared between different groups using Kaplan-Meier (KM) method and a log-rank test. Wilcoxon's signed rank test was used to compare the difference of infiltration scores between the PDL2-high group and the PDL2-low group in each ICI cohort. $P$ value of $<0.05$ was considered to indicate statistical significance in these analyses. All statistical analyses were performed on $\mathrm{R}$ version 4.0.0.

\section{Results}

3.1. PDL2 Is Associated with an Activated Antitumor Environment in TCGA. To investigate the possible effect of PDL2 on immune cell infiltration, GSEA was performed in TCGA pooled cohort. As shown in Figures 1(a)-1(c), CD8 $+\mathrm{T}$ cell signatures $(\mathrm{NES}=1.82, P=0.0083)$, DC signature $(\mathrm{NES}=2.44, P<0.0001)$, and Th1 signature $(\mathrm{NES}=2.77, P$ $<0.0001)$ were significantly enriched in the high PDL2 expression group. To evaluate the differences between different tumor types, we performed GSEA in 32 separate cancers (Figures S1-S3). Although NES was slightly different, CD8+ $\mathrm{T}$ cell signature and DC signature were enriched in the PDL2-high group in most cancer types (Figures 1(d) and 1(e)). Th1 cell signatures of most cancers were enriched in the PDL2-high group, but in the THYM group, the PDL2low group showed more intense Th1 cell signatures than the PDL2-high group. Generally, PDL2 expression is associated with CD8+ T cell, DC, and Th1 cell infiltration, which critically operate in cancer immunotherapy.

Suppressed $\mathrm{T}$ cell activation is crucial to the tumor immune escape mechanisms; we collected three IFN- $\gamma$ signatures to assess the level of T cell activation: Louis signature $(\mathrm{NES}=1.85, P<0.0001)$, Mark signature $(\mathrm{NES}=2.07, P<$ $0.0001)$, and Padmanee signature (NES $=2.10, P<0.0001)$, which were significantly enriched in the PDL2-high group, suggesting that stronger $\mathrm{T}$ cell activation may exist in patients of the PDL2-high group (Figures 1(g)-1(i)). Individual analysis was performed (Figures S4-S6). Three IFN$\gamma$ signatures tend to be enriched in the PDL2-high group among cancers (Figures 1(g)-1(i)): DLBC, LUSC, COAD, SARC, and TGCT were the leading cancers with the highest NES by the Louis signature analysis (Figure 1(j)). By the Mark signature and Padmanee signature analysis, LUSC, READ, LUAD, HCC, and COAD were detected as the top 5 NES rankings, but Mark signature rankings were LUSC, COAD, LUAD, READ, and HCC; Padmanee signature ranking was READ, LUSC, HCC, LUAD, and COAD (Figures 1(k) and 1(l)). Generally, the PDL2-high group showed a more intense IFN- $\gamma$ signature than the PDL2-low group. Better antigen presentation, $\mathrm{T}$ cell activation, and stronger cytotoxicity may exist in the PDL2-high group.

3.2. Association of PDL2 Expression with Prognosis of ICI Treatment. Since PDL2 was related to the antitumor 


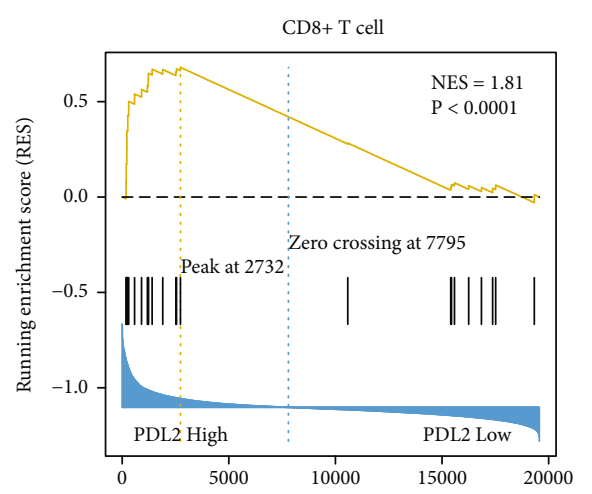

(a)

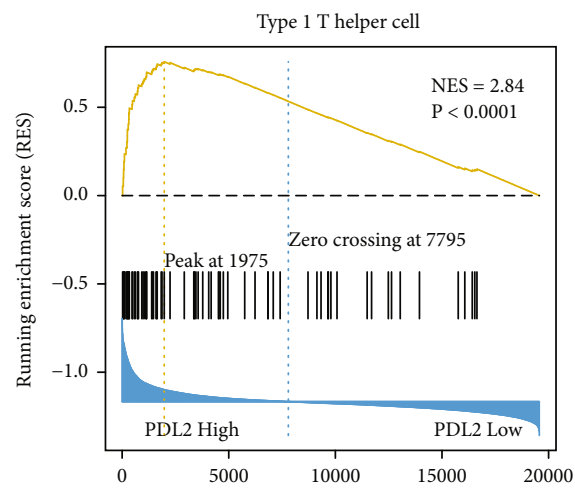

(c)

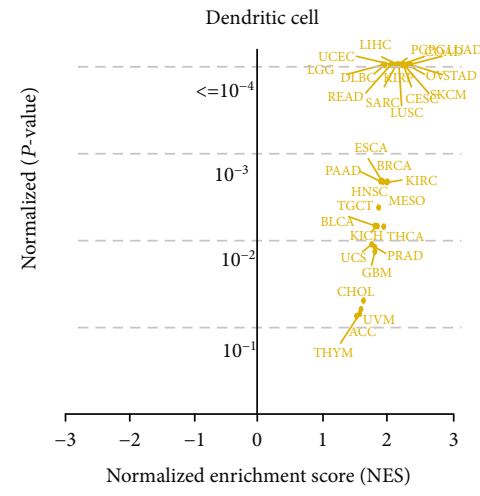

(e)

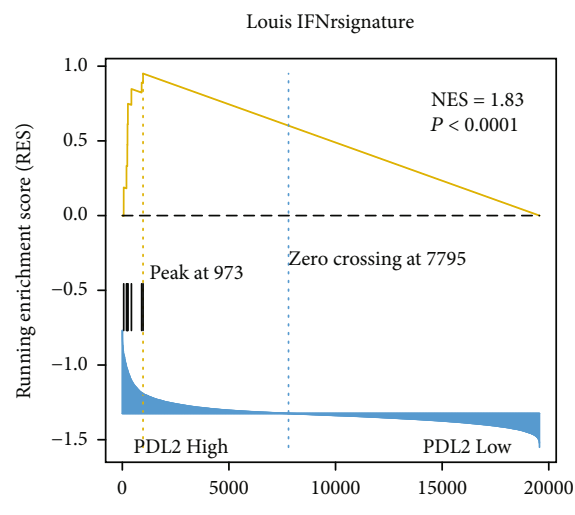

(g)

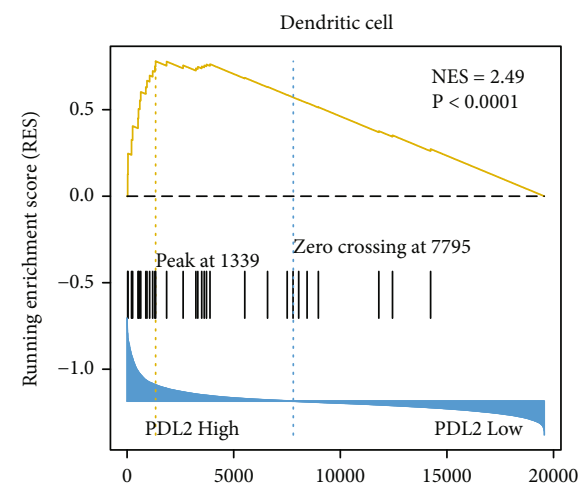

(b)

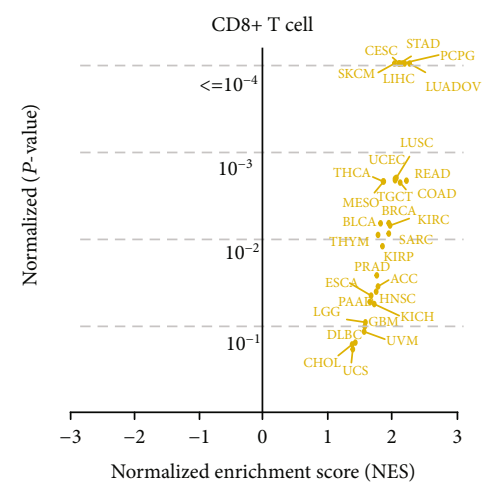

(d)

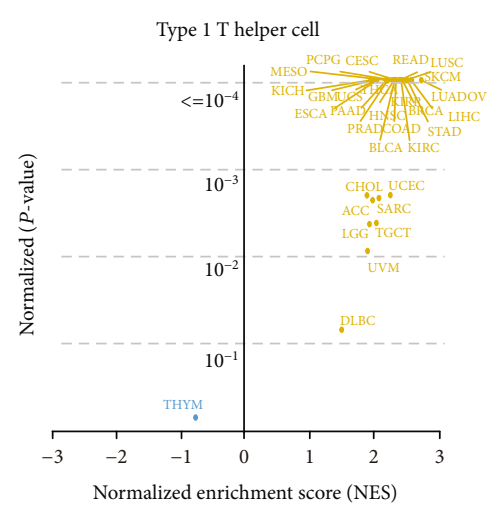

(f)

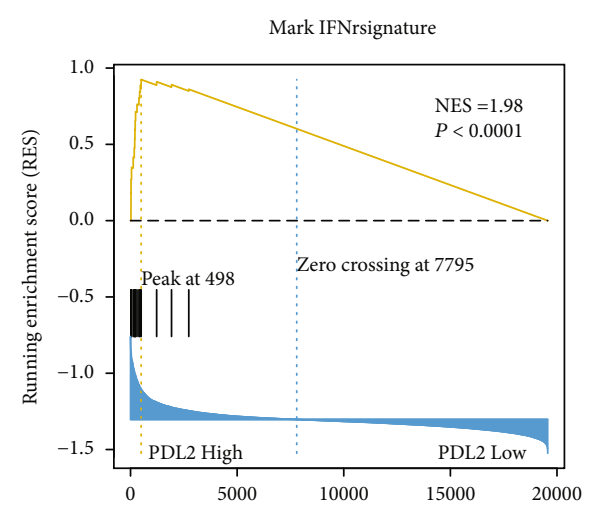

(h)

Figure 1: Continued. 


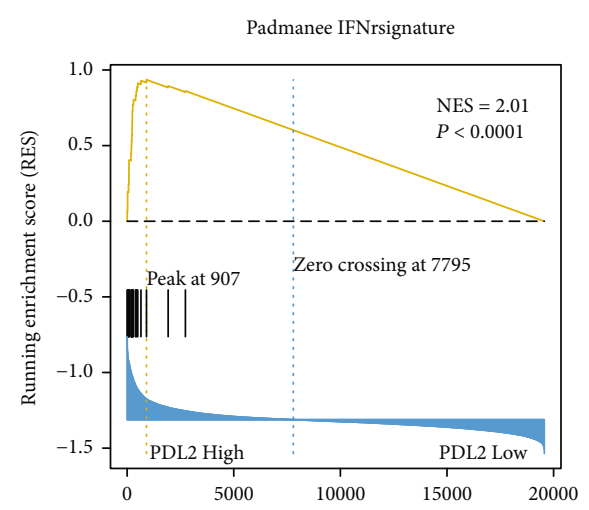

(i)

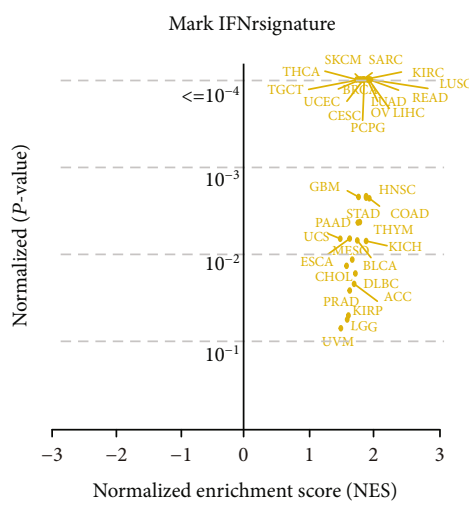

(k)

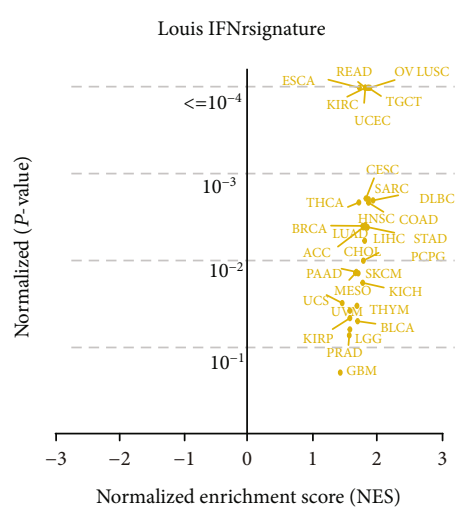

(j)

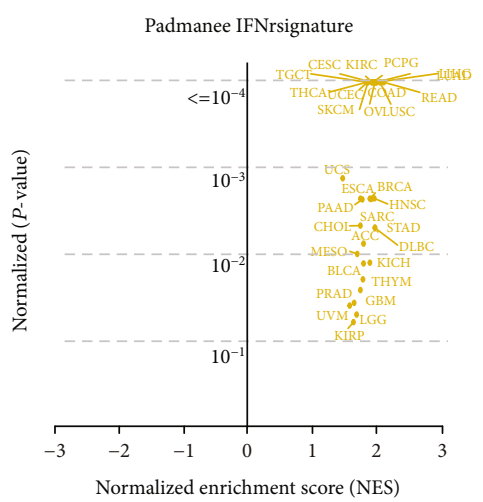

(1)

FIGURE 1: Enrichment and summary plots of the immune-related signatures in TCGA. Enrichment plots of (a) CD8+ cell signature, (b) dendritic cell signature, and (c) Th1 signature in TCGA pooled cohort, all cell signatures of the PDL2-high group were mostly enriched. (d, e) Summary plots of cell signatures with enrichment NES and $P$ values in each tumor type, except for the Th1 signature enrichment in the PDL2-low group of THYM, all results are consistent with the results of TCGA pooled cohort. (g-i) Enrichment plots with IFN- $\gamma$ signatures in TCGA pooled cohort, all IFN- $\gamma$ signatures were enriched in the PDL2-high group. (j-l) Summary plots of IFN- $\gamma$ signatures with enrichment NES and $P$ value in each tumor type, all IFN- $\gamma$ signatures were enriched in the PDL2-high group.

immune environment, we first aimed to determine whether PDL2 affects the prognosis of ICI treatment. Higher response rate of the PDL2-high group was observed in the Gide cohort, Liu cohort, Mariathasan cohort, and pooled cohort (Figure 2(a)). In the pooled cohort, the response rate of the PDL2-high group was $53.6 \%$ compared to $39.4 \%$ in the PDL2-low group (Figure 2(a)). Consistent with the result of response rate, high expression of PDL2 is a positive factor for survival in the ICI cohort (Figures 2(b) and 2(c)). The patients in the PDL2-high group experienced longer OS than those in the PDL2-low group when analyzing in the Liu cohort, Miao cohort, and pooled cohort (Figures 2(e), 2(g), and 2(i); Liu: $\operatorname{HR}[95 \% \mathrm{CI}]=0.58[0.35-0.96], \quad P=0.032$; Miao: $\operatorname{HR}[95 \% \mathrm{CI}]=0.28[0.10-0.77], \quad P=0.010$; pooled: $\mathrm{HR}[95 \% \mathrm{CI}]=0.71[0.57-0.87], P=0.001)$. Longer OS was also seen in patients in the PDL2-high group in the Gide cohort, Mariathasan cohort, and Van Allen cohort; however, those results were not statistically significant (Figures 2(d), 2(f), and 2(h); Gide: $\operatorname{HR}[95 \% \mathrm{CI}]=0.52[0.24-1.12], P=$ 0.091; Mariathasan: $\operatorname{HR}[95 \% \mathrm{CI}]=[0.63-1.06], P=0.124$; Van Allen: HR $[95 \% \mathrm{CI}]=[0.25-1.16], P=0.108)$.

We next investigated the effect of PDL2 expression on non-ICI treatment prognosis in TCGA database. We found that there is no significant difference in OS between the PDL2-high group and the PDL2-low group in TCGA pooled cohort (Figure S7, HR [95\%CI] $=0.98[0.90-1.07], P=0.603$ ), suggesting that higher PDL2 expression was not a prognostic factor in non-ICI treatment.

3.3. Verifying Association between PDL2 and TME in ICI Cohorts. We explored the relationship between PDL2 and TME in the ICI-treated cohorts. We first evaluated the expression of Padmanee IFN- $\gamma$ immune genes and PDL2 (Figure 3(a)). Thereafter, the immune cell infiltration of each sample was quantified by ssGSEA in the ICI cohorts. In the Gide cohort, the ssGSEA scores of CD8+ T cell, DC, and Th1 cells were higher in the PDL2-high group than the scores in the PDL2-low group (Figure 3(b)), and this is consistent with the results of the analysis of other melanoma cohorts (Figures 3(c) and 3(f)). In bladder cancer and the Mariathasan cohort, higher ssGSEA scores were observed in the PDL2-high group (Figure 3(d)). In the Miao cohort, $\mathrm{T}$ cell infiltration and Th1 cell infiltration were still significantly higher in the PDL2-high group; the DC infiltration trends were also higher in the PDL2-high group, but not significantly (Figure $3(\mathrm{e})$ ). These results suggest that high 


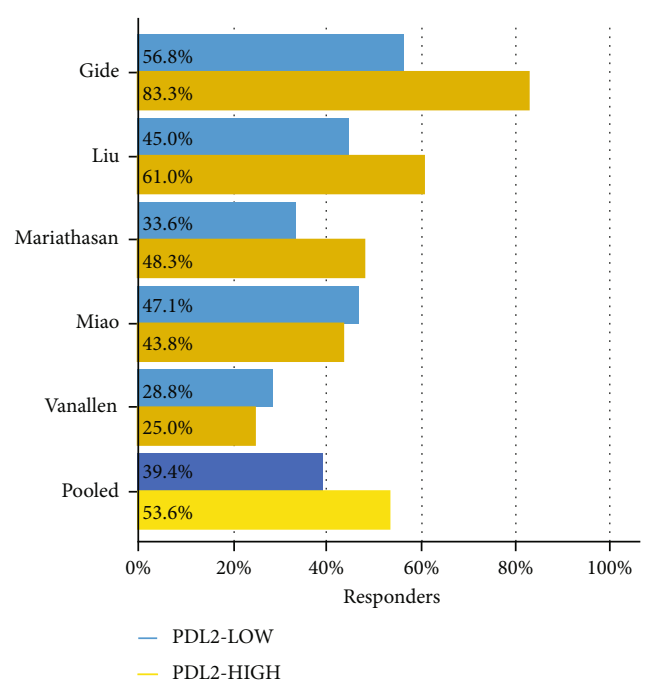

(a)

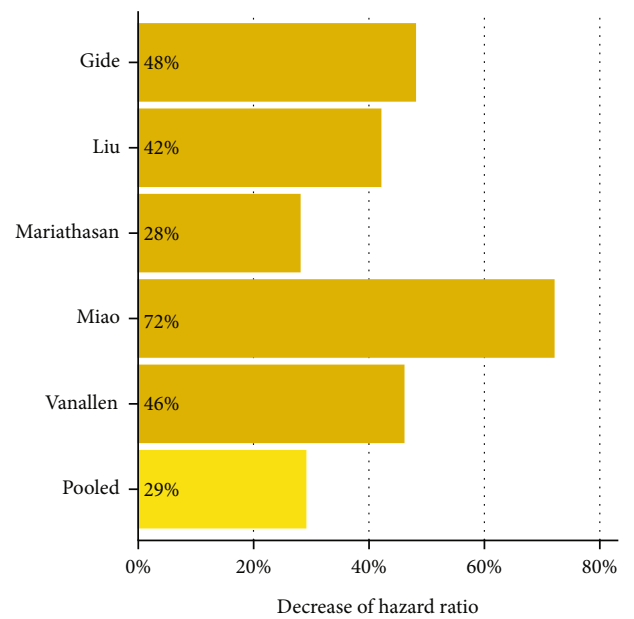

(c)

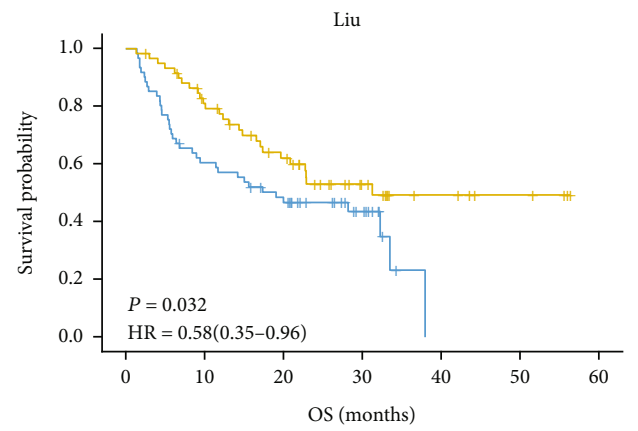

No.at Risk

$\begin{array}{lllllllll}\text { LOW } & 61 & 36 & 27 & 12 & 0 & 0 & 0\end{array}$

$\begin{array}{llllllll}\text { HIGH } & 60 & 45 & 31 & 15 & 7 & 4 & 0\end{array}$

- PDL2-LOW

— PDL2-HIGH
Cohort

Pts $\quad$ HR $(95 \% \mathrm{CL}) \quad P$-value

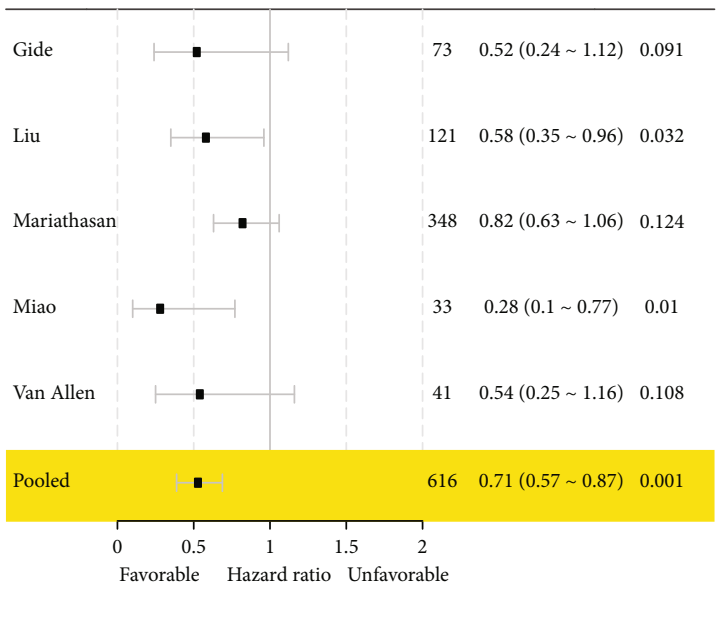

(b)

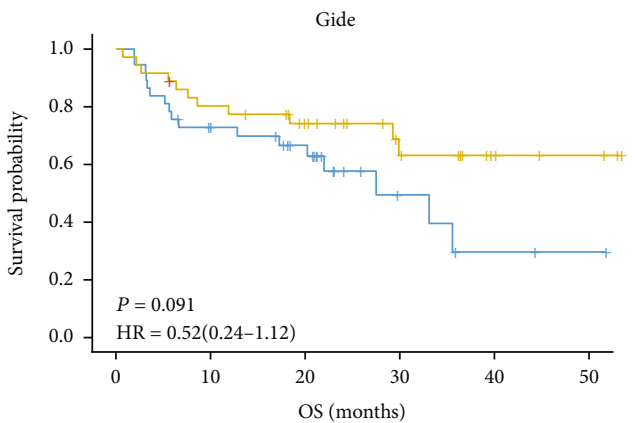

No.at Risk

$\begin{array}{lllllll}\text { LOW } & 37 & 25 & 18 & 5 & 2 & 1\end{array}$

HIGH $\begin{array}{llllll}36 & 28 & 21 & 11 & 5 & 3\end{array}$

- PDL2-LOW

- PDL2-HIGH

(d)

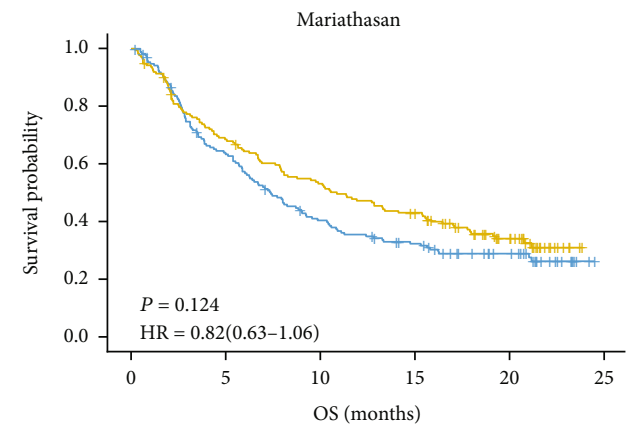

No.at Risk

$\begin{array}{lllllll}\text { LOW } & 174 & 106 & 66 & 49 & 30 & 0\end{array}$

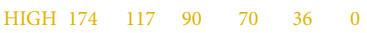

- PDL2-LOW

— PDL2-HIGH

Figure 2: Continued. 


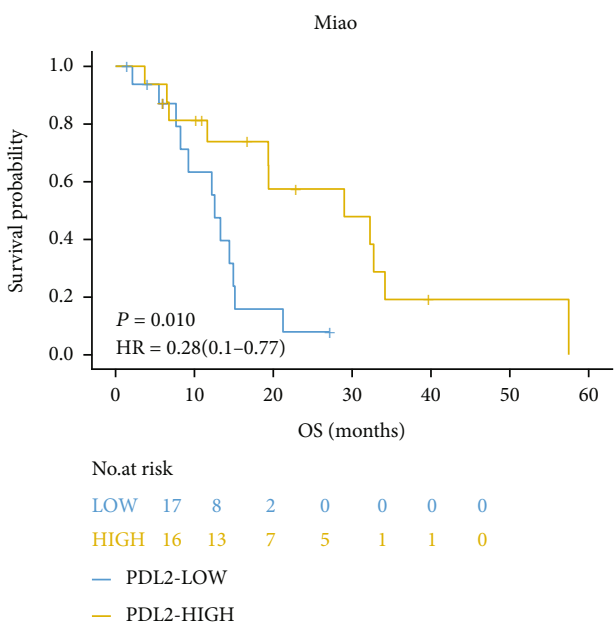

(g)

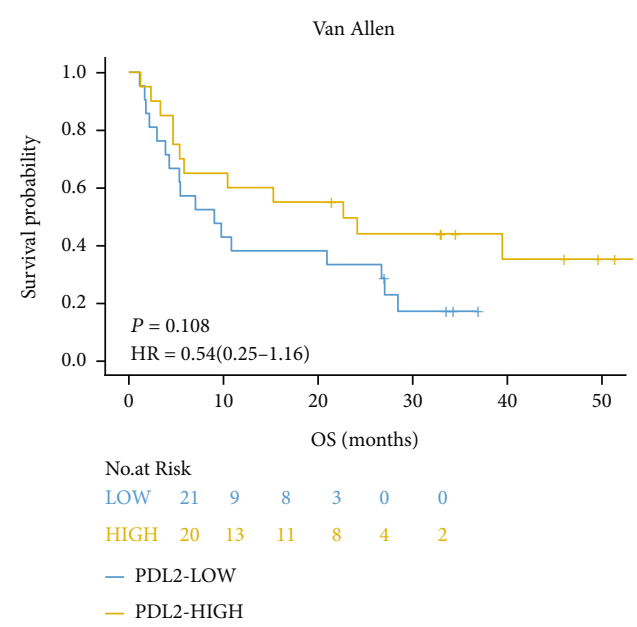

(h)

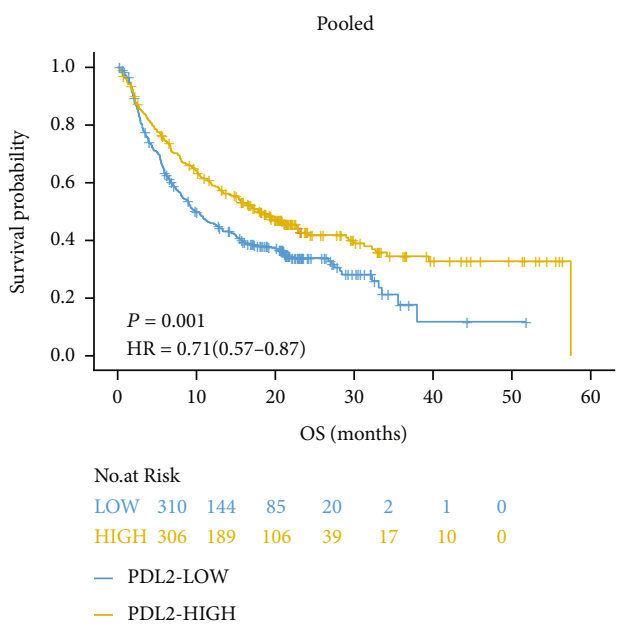

(i)

FIGURE 2: Survival analysis between the PDL2-high group and the PDL2-low group in the ICI cohorts. (a) Histogram describing proportions of responders in different groups of ICI cohorts; except the Miao cohort and Van Allen cohort, the PDL2-high group has more responders than the PDL2-low group. (b) Univariate Cox analysis according to PDL2 expression median in the ICI cohorts; high PDL2 expression is protective in ICI-treated patients. (c) The percentage decrease of HR caused by high PDL2 expression in the ICI cohort. (d-i) KM plot of OS in ICI cohorts comparing patients with high and low PDL2 expressions and longer OS were observed in the PDL2-high group.

expression of PDL2 is accompanied by an antitumor immune environment in the ICI cohorts.

\section{Discussion}

This study investigated the potential use of PDL2 expression as a prognostic marker of the effect of ICI treatment by integrating published RNA-seq with clinical data. Our study indicated that high expression of PDL2 is associated with high rates of response to treatment by ICIs and long OS for patients across multiple cancer types. The immune cell analysis indicated that higher expression of PDL2 may be accompanied with higher level of CD8+ T cell, DC, and Th1 cell infiltration; additionally, IFN-r signature analysis suggests better $\mathrm{T}$ cell activation may occur in patients with high PDL2 expression. Our study is one of the first to pro- pose PDL2 as a predictive biomarker for analyzing responses to ICI treatment across multiple cancers.

CD8+ T cells directly participate in the killing of tumor cells in TME [34]. PDL1 is expressed by tumor cells to resist the antitumor immunity mediated by CD8+ T cells, which prevents $\mathrm{T}$ cell activation [9]. This expression is induced by the mutations of tumor cells and the IFN- $\gamma$ secreted by $\mathrm{T}$ cells [35]. IFN- $\gamma$ from $\mathrm{T}$ cells is a major factor for most cancers [36]. PDL1 expression usually reflects the high IFN- $\gamma$ secreted in the TME, which indicates the strong antitumor immunity, such as CD8+ T cell response. Thus, PDL1 is a predictive marker of ICI treatment. However, in some clinical experiments, high PDL1 expression was not associated with the clinical effect of ICI treatment, and PDL1negative patients benefited from ICI treatment, suggesting that PDL1 cannot fully represent the antitumor immunity [10-12]. PDL2 is another ligand that received less research 

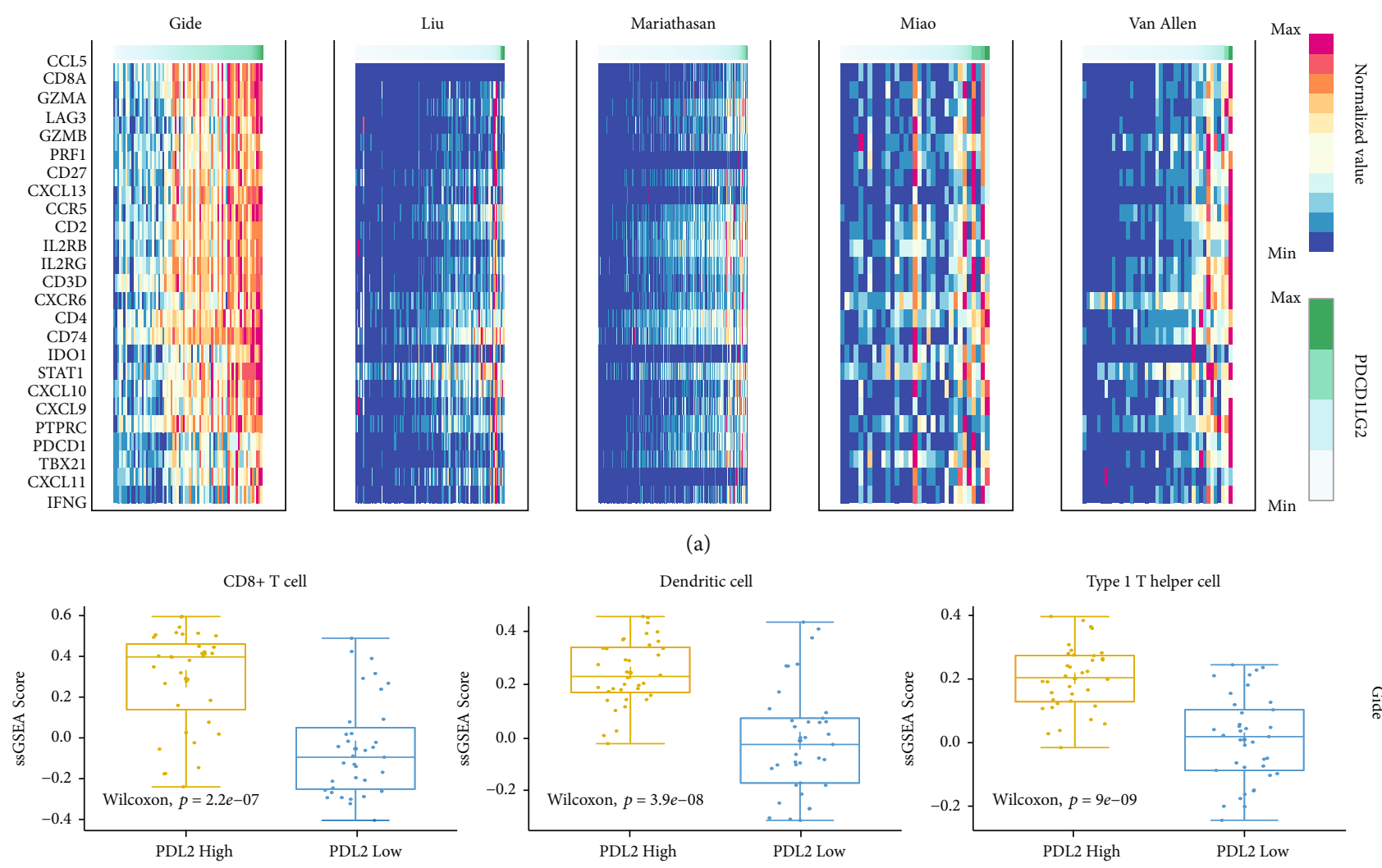

(b)
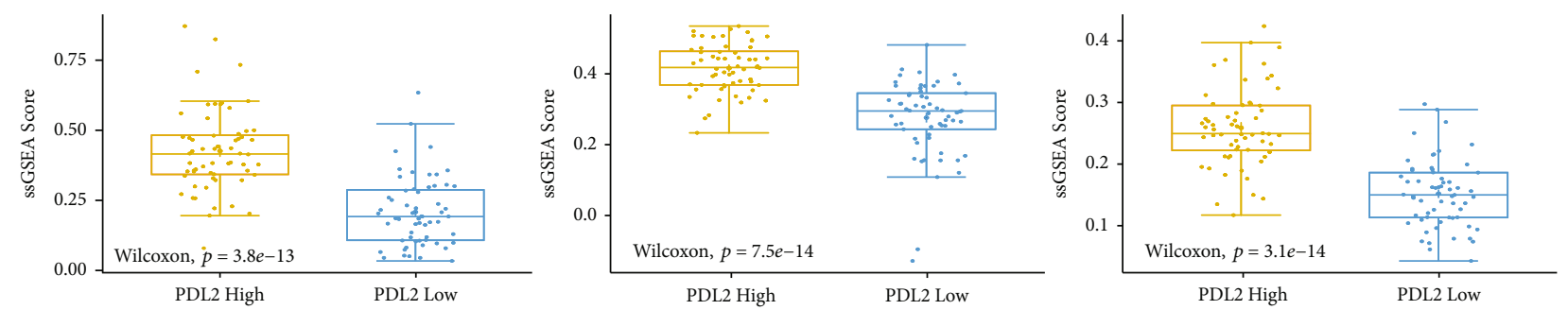

(c)
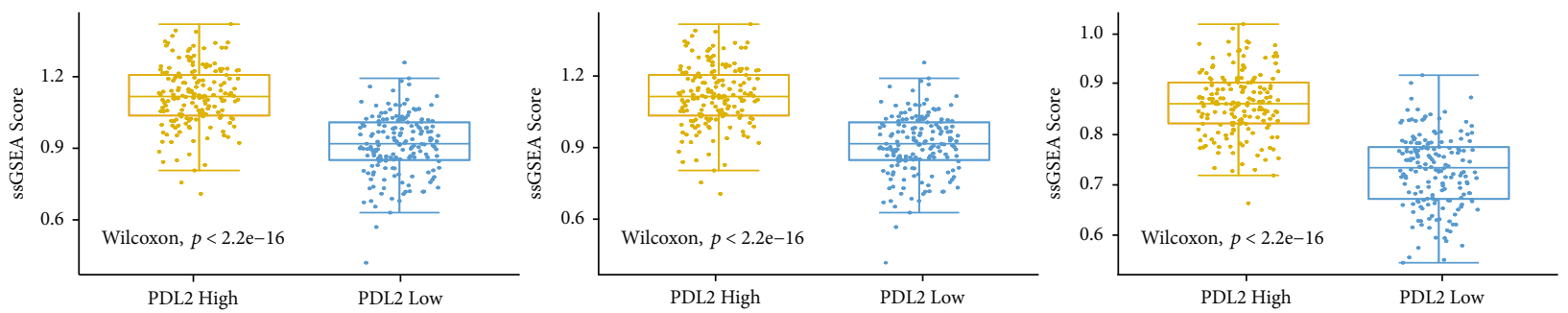

(d)
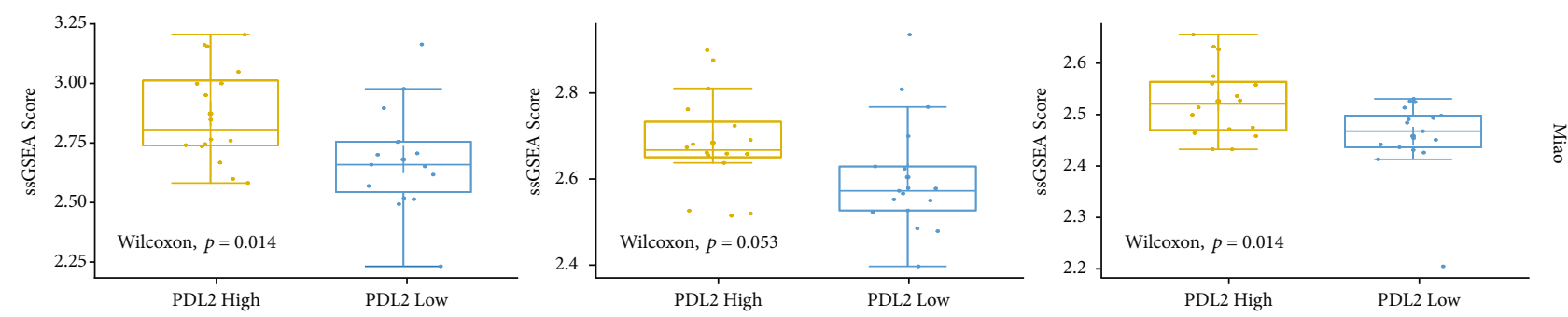

(e)

Figure 3: Continued. 

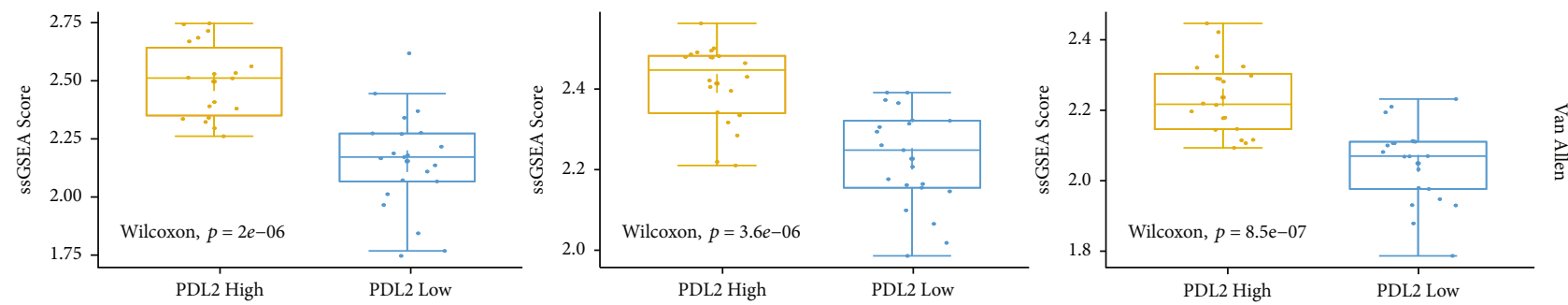

(f)

FIgure 3: Association between PDL2 expression and immune-related signatures in the ICI cohorts. (a) Heat map of Padmanee IFN- $\gamma$ signature genes in ICI-treated cohorts. (b-f) The ssGSEA score of immune cell signatures comparing between the PDL2-high group and the PDL2-low group in the ICI-treated cohorts. Wilcoxon's test was used to compare the differences.

attention in the early stages. Recent evidence proves that PDL2 is closely associated with the TME [23]. The IHC results of PDL2 in different cancers were inconsistent with the IHC results of PDL1, in which PDL2-positive IHC and PDL1-negative IHC patients responded to the ICI treatment [21]. Animal models showed overexpression of PDL2, which can induce a rapid tumor proliferation, and anti-PDL2 treatment could eradicate the resistance to anti-PDL1 drugs [23]. Our results are in agreement with the above research, and we found that high expression of PDL2 is associated with an activated antitumor environment in both TCGA database and the IO cohorts. The expression of PDL2 could be an evidence of ICI treatment.

In previous research, the expression of PDL2 was induced by the NF- $\kappa \mathrm{B}$ pathway and the signal transducer and activator of transcription- (STAT-) 6 pathway [37]. Knockout NF- $\kappa$ B cannot eliminate PDL2 expression [38]. After IFN- $\gamma$ and LPS stimulation, DCs from NF- $\kappa \mathrm{B}$ $\mathrm{p} 50^{-1-} \mathrm{p} 65^{-1+}$ mice were incapable of upregulating PDL2 expression [38]. However, another study showed that NF- $\kappa \mathrm{B}$ p $50^{-1-}$ mice cannot produce IL- 4 and IL-13, the activators of STAT-6 [39]. STAT-6 is an important transcription factor and signal transduction molecule for many biological processes, especially for the Th2 immune responses [40]. As it is induced by NF- $\kappa \mathrm{B}$ and STAT-6, PDL2 may be involved in the Th2 immune response [37]. However, in our research, PDL2 expression was strongly correlated with Th1 cell infiltration which may be because PDL2 is coexpressed with PDL1. This was investigated to reveal that PDL2 participates in the Th1 immune response. However, more investigation is needed to clarify how PDL2 affects the TME.

This research has some limitations, the limited sample size in one ICI-treated cohort that may have introduced statistical bias. However, consistent results from numerous cohorts may have minimized the bias. Secondly, the availability of limited information prevented us from verifying the influences of functional PDL2 mutation on the prognosis of ICI treatment. Considering that the IHC results of PDL2 could provide a clear cut-off value, an investigation on the PDL2 mutation may clarify the immune functions of PDL2. More molecular studies involving animal models and cell lines are needed. Thirdly, the objective response rate (ORR) of many ICI-treated patients was not evaluated, which may have weakened the statistical analysis; however, the pooled cohort analysis has minimized such biases.

\section{Conclusions}

In summary, our results indicated that PDL2 was associated with an antitumor effect and can be a potential predictive biomarker to screen patients' benefit from ICI treatment in multiple cancers.

\section{Abbreviations}

ACC: Adrenocortical carcinoma

BLCA: Bladder urothelial carcinoma

BRCA: Breast invasive carcinoma

CESC: Cervical squamous cell carcinoma and endocervical adenocarcinoma

CHOL: Cholangiocarcinoma

COAD: Colon adenocarcinoma

DLBC: Lymphoid neoplasm diffuse large B cell lymphoma

ESCA: Esophageal carcinoma

GBM: Glioblastoma multiforme

HNSC: Head and neck squamous cell carcinoma

KICH: Kidney chromophobe

KIRC: Kidney renal clear cell carcinoma

KIRP: Kidney renal papillary cell carcinoma

LAML: Acute myeloid leukemia

LGG: Brain lower grade glioma

LIHC: Liver hepatocellular carcinoma

LUAD: Lung adenocarcinoma

LUSC: Lung squamous cell carcinoma

MESO: Mesothelioma

OV: $\quad$ Ovarian serous cystadenocarcinoma

PAAD: Pancreatic adenocarcinoma

PCPG: Pheochromocytoma and paraganglioma

PRAD: Prostate adenocarcinoma

READ: Rectum adenocarcinoma

SARC: Sarcoma

SKCM: Skin cutaneous melanoma

STAD: Stomach adenocarcinoma

TGCT: Testicular germ cell tumors

THCA: Thyroid carcinoma

THYM: Thymoma

UCEC: Uterine corpus endometrial carcinoma

UCS: Uterine carcinosarcoma

UVM: Uveal melanoma. 


\section{Data Availability}

TCGA data: the data were downloaded from The Cancer Genome Atlas (TCGA) data portal (https://portal.gdc.cancer .gov/) though $\mathrm{R}$ package TCGAbiolinks (https:// bioconductor.org/packages/release/bioc/html/TCGAbiolinks .html). Gide cohort data are available from NCBI Sequence Read Archive (SRA) repository under project no. PRJEB23709. Liu cohort data and Miao cohort data are collected from supplementary materials of original article (Liu: https://www .nature.com/articles/s41591-019-0654-5\#Sec32; Miao: "https:// www.ncbi.nlm.nih.gov/pmc/articles/PMC6035749/"). Mariathasan cohort data are collected from R package "Imvigor 210" (http://research-pub.gene.com/IMvigor210CoreBiologies/ ). Van Allen cohort data are public in github (https://github .com/vanallenlab/VanAllen_CTLA4_Science_RNASeq_TPM).

\section{Conflicts of Interest}

The authors declare that there is no conflict of interest regarding the publication of this paper.

\section{Authors' Contributions}

QJ and BZ conceived/designed the work. AW, HC, and ZJ acquired/analyzed/interpreted the data. AW and HC drafted the manuscript. AW, HC, and ZJ prepared the figures. AW, HC, ZJ, QJ, and BZ reviewed the manuscript. All authors read and approved the final manuscript. Aoyun Wang and Han Chu contributed equally to this work.

\section{Acknowledgments}

We thank all the $\mathrm{R}$ programming package developers.

\section{Supplementary Materials}

Figure S1: enrichment plots of the CD8+ T cell signatures in TCGA. Figure S2: enrichment plots of the dendritic cell signatures in TCGA. Figure S3: enrichment plots of the type 1 helper cell signatures in TCGA. Figure S4: enrichment plots of the Louis IFN- $\gamma$ signatures in TCGA. Figure S5: enrichment plots of the Mark IFN- $\gamma$ signatures in TCGA. Figure S6: enrichment plots of the Padmanee IFN- $\gamma$ signatures in TCGA. Figure S7: survival analysis of PDL2 in TCGA pooled cohort. (Supplementary Materials)

\section{References}

[1] J. R. Brahmer, C. G. Drake, I. Wollner et al., "Phase I study of single-agent anti-programmed death-1 (MDX-1106) in refractory solid tumors: safety, clinical activity, pharmacodynamics, and immunologic correlates," Journal of Clinical Oncology, vol. 28, no. 19, pp. 3167-3175, 2010.

[2] F. S. Hodi, M. C. Mihm, R. J. Soiffer et al., "Biologic activity of cytotoxic T lymphocyte-associated antigen 4 antibody blockade in previously vaccinated metastatic melanoma and ovarian carcinoma patients," Proceedings of the National Academy of Sciences of the United States of America, vol. 100, no. 8, pp. 4712-4717, 2003.
[3] A. Ribas, L. H. Camacho, G. Lopez-Berestein et al., "Antitumor activity in melanoma and anti-self responses in a phase I trial with the anti-cytotoxic $\mathrm{T}$ lymphocyte-associated antigen 4 monoclonal antibody CP-675,206," Journal of Clinical Oncology, vol. 23, no. 35, pp. 8968-8977, 2005.

[4] S. L. Topalian, F. S. Hodi, J. R. Brahmer et al., "Safety, activity, and immune correlates of anti-PD-1 antibody in cancer," The New England Journal of Medicine, vol. 366, no. 26, pp. 24432454, 2012.

[5] G. J. Freeman, A. J. Long, Y. Iwai et al., "Engagement of the PD-1 immunoinhibitory receptor by a novel B7 family member leads to negative regulation of lymphocyte activation," The Journal of Experimental Medicine, vol. 192, no. 7, pp. 1027-1034, 2000.

[6] Y. Latchman, C. R. Wood, T. Chernova et al., "PD-L2 is a second ligand for PD-1 and inhibits T cell activation," Nature Immunology, vol. 2, no. 3, pp. 261-268, 2001.

[7] E. Hui, J. Cheung, J. Zhu et al., "T cell costimulatory receptor CD28 is a primary target for PD-1-mediated inhibition," Science, vol. 355, no. 6332, pp. 1428-1433, 2017.

[8] A. O. Kamphorst, A. Wieland, T. Nasti et al., "Rescue of exhausted CD8 T cells by PD-1-targeted therapies is CD28dependent," Science, vol. 355, no. 6332, pp. 1423-1427, 2017.

[9] T. J. Curiel, S. Wei, H. Dong et al., "Blockade of B7-H1 improves myeloid dendritic cell-mediated antitumor immunity," Nature Medicine, vol. 9, no. 5, pp. 562-567, 2003.

[10] E. B. Garon, N. A. Rizvi, R. Hui et al., "Pembrolizumab for the treatment of non-small-cell lung cancer," The New England Journal of Medicine, vol. 372, no. 21, pp. 2018-2028, 2015.

[11] C. Robert, G. V. Long, B. Brady et al., "Nivolumab in previously untreated melanoma withoutBRAFMutation," The New England Journal of Medicine, vol. 372, no. 4, pp. 320-330, 2015.

[12] C. Robert, J. Schachter, G. V. Long et al., "Pembrolizumab versus ipilimumab in advanced melanoma," The New England Journal of Medicine, vol. 372, no. 26, pp. 2521-2532, 2015.

[13] J. Brahmer, K. L. Reckamp, P. Baas et al., "Nivolumab versus docetaxel in advanced squamous-cell non-small-cell lung cancer," The New England Journal of Medicine, vol. 373, no. 2, pp. 123-135, 2015.

[14] Y.-K. Kang, N. Boku, T. Satoh et al., "Nivolumab in patients with advanced gastric or gastro-oesophageal junction cancer refractory to, or intolerant of, at least two previous chemotherapy regimens (ONO-4538-12, ATTRACTION-2): a randomised, double-blind, placebo- controlled, phase 3 trial," Lancet, vol. 390, no. 10111, pp. 2461-2471, 2017.

[15] R. J. Motzer, B. Escudier, D. F. McDermott et al., "Nivolumab versus everolimus in advanced renal-cell carcinoma," The New England Journal of Medicine, vol. 373, no. 19, pp. 1803-1813, 2015.

[16] A. L. Kinter, E. J. Godbout, J. P. McNally et al., "The common gamma-chain cytokines IL-2, IL-7, IL-15, and IL-21 induce the expression of programmed death-1 and its ligands," Journal of Immunology, vol. 181, no. 10, pp. 6738-6746, 2008.

[17] W. J. Lesterhuis, C. J. A. Punt, S. V. Hato et al., "Platinumbased drugs disrupt STAT6-mediated suppression of immune responses against cancer in humans and mice," The Journal of Clinical Investigation, vol. 121, no. 8, pp. 3100-3108, 2011.

[18] N. Messal, N. E. Serriari, S. Pastor, J. A. Nunes, and D. Olive, "PD-L2 is expressed on activated human T cells and regulates 
their function," Molecular Immunology, vol. 48, no. 15-16, pp. 2214-2219, 2011.

[19] X. Zhong, J. R. Tumang, W. Gao, C. Bai, and T. L. Rothstein, "PD-L2 expression extends beyond dendritic cells/macrophages to B1 cells enriched for $\mathrm{V}(\mathrm{H}) 11 / \mathrm{V}(\mathrm{H}) 12$ and phosphatidylcholine binding," European Journal of Immunology, vol. 37, no. 9, pp. 2405-2410, 2007.

[20] S. G. Zhao, J. Lehrer, S. L. Chang et al., "The immune landscape of prostate cancer and nomination of PD-L2 as a potential therapeutic target," Journal of the National Cancer Institute, vol. 111, no. 3, pp. 301-310, 2019.

[21] J. H. Yearley, C. Gibson, N. Yu et al., "PD-L2 expression in human tumors: relevance to anti-PD-1 therapy in cancer," Clinical Cancer Research, vol. 23, no. 12, pp. 3158-3167, 2017.

[22] Y. R. Miao, K. N. Thakkar, J. Qian et al., "Neutralization of PDL2 is essential for overcoming immune checkpoint blockade resistance in ovarian cancer," Clinical Cancer Research, vol. 27, no. 15, pp. 4435-4448, 2021.

[23] T. Tanegashima, Y. Togashi, K. Azuma et al., "Immune suppression by PD-L2 against spontaneous and treatmentrelated antitumor immunity," Clinical Cancer Research, vol. 25, no. 15, pp. 4808-4819, 2019.

[24] S. M. Ahmad, E. Martinenaite, M. Holmström et al., "The inhibitory checkpoint, PD-L2, is a target for effector T cells: novel possibilities for immune therapy," Oncoimmunology, vol. 7, no. 2, p. e1390641, 2018.

[25] T. N. Gide, C. Quek, A. M. Menzies et al., "Distinct immune cell populations define response to anti-PD-1 monotherapy and anti-PD-1/anti-CTLA-4 combined therapy," Cancer Cell, vol. 35, no. 2, pp. 238-255.e6, 2019.

[26] D. Liu, B. Schilling, D. Liu et al., "Integrative molecular and clinical modeling of clinical outcomes to PD1 blockade in patients with metastatic melanoma," Nature Medicine, vol. 25, no. 12, pp. 1916-1927, 2019.

[27] S. Mariathasan, S. J. Turley, D. Nickles et al., "TGF $\beta$ attenuates tumour response to PD-L1 blockade by contributing to exclusion of T cells," Nature, vol. 554, no. 7693, pp. 544-548, 2018.

[28] D. Miao, C. A. Margolis, W. Gao et al., "Genomic correlates of response to immune checkpoint therapies in clear cell renal cell carcinoma," Science, vol. 359, no. 6377, pp. 801-806, 2018.

[29] E. M. Van Allen, D. Miao, B. Schilling et al., "Genomic correlates of response to CTLA-4 blockade in metastatic melanoma," Science, vol. 350, no. 6257, pp. 207-211, 2015.

[30] M. Ayers, J. Lunceford, M. Nebozhyn et al., "IFN- $\gamma$-related mRNA profile predicts clinical response to PD-1 blockade," The Journal of Clinical Investigation, vol. 127, no. 8, pp. 2930-2940, 2017.

[31] P. Charoentong, F. Finotello, M. Angelova et al., "Pan-cancer immunogenomic analyses reveal genotypeimmunophenotype relationships and predictors of response to checkpoint blockade," Cell Reports, vol. 18, no. 1, pp. 248262, 2017.

[32] L. Fehrenbacher, A. Spira, M. Ballinger et al., “Atezolizumab versus docetaxel for patients with previously treated nonsmall- cell lung cancer (POPLAR): a multicentre, open-label, phase 2 randomised controlled trial," Lancet, vol. 387, no. 10030, pp. 1837-1846, 2016.

[33] P. Sharma, M. Retz, A. Siefker-Radtke et al., "Nivolumab in metastatic urothelial carcinoma after platinum therapy (CheckMate 275): a multicentre, single-arm, phase 2 trial," The Lancet Oncology, vol. 18, no. 3, pp. 312-322, 2017.
[34] C. Fu and A. Jiang, "Dendritic cells and CD8 T cell immunity in tumor microenvironment," Frontiers in Immunology, vol. 9, 2018.

[35] S. L. Topalian, C. G. Drake, and D. M. Pardoll, "Immune checkpoint blockade: a common denominator approach to cancer therapy," Cancer Cell, vol. 27, no. 4, pp. 450-461, 2015.

[36] T. Powles, J. P. Eder, G. D. Fine et al., "MPDL3280A (anti-PDL1) treatment leads to clinical activity in metastatic bladder cancer," Nature, vol. 515, no. 7528, pp. 558-562, 2014.

[37] E. N. Rozali, S. V. Hato, B. W. Robinson, R. A. Lake, and W. J. Lesterhuis, "Programmed death ligand 2 in cancer-induced immune suppression," Clinical and Developmental Immunology, vol. 2012, Article ID 656340, 8 pages, 2012.

[38] S. . C. Liang, Y. . E. Latchman, J. . E. Buhlmann et al., "Regulation of PD-1, PD-L1, and PD-L2 expression during normal and autoimmune responses," European Journal of Immunology, vol. 33, no. 10, pp. 2706-2716, 2003.

[39] J. Das, C.-H. Chen, L. Yang, L. Cohn, P. Ray, and A. Ray, “A critical role for NF- $\kappa$ B in _Gata3_expression and $\mathrm{T}_{\mathrm{H}} 2$ differentiation in allergic airway inflammation," Nature Immunology, vol. 2, no. 1, pp. 45-50, 2001.

[40] D. Hebenstreit, G. Wirnsberger, J. Horejs-Hoeck, and A. Duschl, "Signaling mechanisms, interaction partners, and target genes of STAT6," Cytokine \& Growth Factor Reviews, vol. 17 , no. 3, pp. 173-188, 2006. 